\title{
Is it safe to perform myomectomy in first trimester of pregnancy? The report of two cases
}

\author{
Murat Celiloglu ${ }^{1}$, Samican Ozmen ${ }^{1 *}$, Sefa Kurt ${ }^{1}$, Orkun Ilgen ${ }^{1}$ \\ ${ }^{1}$ DOKUZ EYLUL UNIVERSITY SCHOOL OF MEDICINE, DEPARTMENT OF OBSTETRICS AND GYNECOLOGY, IZMIR, TURKEY
}

\begin{abstract}
Uterine fibroids are the most common benign tumors in women in the reproductive age group. The incidence of fibroids during pregnancy varies between 1.6 and $10.7 \%$. In $10 \%$ of these cases, fibroids lead to complications.

The management in symptomatic patients is conservative, and the surgical treatment is delayed until after birth due to its risks. In the last two decades, it has been shown that myomectomy can be an alternative treatment in selected cases, especially in second-trimester pregnancies. However, the data on the success of myomectomies performed earlier in pregnancy are limited.

In this case report, we present two cases who were admitted to our clinic during the first trimester of pregnancy and had complicated fibroids. The diagnosis, the follow up and the treatment are presented with the review
\end{abstract} of the literature.

\author{
Category: Case Presentation \\ Received: June 06, 2021 \\ Accepted: August 14, 2021 \\ Published: November 15, 2021 \\ Keywords: \\ uterine fibroids, myomectomy, first trimester pregnancy, outcome \\ * Corresponding author: \\ Samican Ozmen, \\ Dokuz Eylul University School of Medicine, Department of \\ Obstetrics and Gynecology, Mithatpasa Cd. No. 1606, 35340 \\ Balcova, Izmir, Turkey \\ E-mail: samicanozmen@hotmail.com
}

\section{Introduction}

Uterine fibroids are the most common benign tumors of the female genital tract [1]. Fibroids are detected in 20-50\% of the women in the reproductive age group. This rate varies according to age, ethnicity and parity [2]. The prevalence of uterine leiomyomas in pregnancy varies between 1.6 and $-10.7 \%[3,4]$. Most of these cases are asymptomatic; however, in $10 \%$ of the cases, uterine fibroids lead to complications [5]. These complications include early pregnancy loss, pelvic pain, placental abruption, hydronephrosis, PPROM, preterm labor, IUGR, fetal mal-presentation and postpartum hemorrhage. Intractable pelvic pain refractory to analgesics is the most common complication related to fibroids. It has been shown that the rate of hospitalization among pregnant patients with uterine leiomyoma who were admitted to the hospital with symptoms of pain and vaginal bleeding was between $5-15 \%$ [6].

Conservative management is the first-line treatment for symptomatic leiomyomas observed during pregnancy, and it includes bed rest, hydration and analgesics. If these treatments fail, performing myomectomy after sharing the possible risks of the procedure with patients, can be an alternative treatment option [7]. The literature consists of studies of successfully performed myomectomies, mainly in the second trimester of pregnancy. In the first trimester, the traditional approach is to induce abortion and surgically remove the fibroid at a later date [8]. Very few studies have shown that, in specific cases, first trimester myomectomies may have similar safety rates and advantages with myomectomies performed in second trimester pregnancies [8].

In this study, two cases of pregnant women with uterine fibroids who were admitted to the hospital with severe abdominal pain refractory to medical treatment and treated with myomectomy are presented.

\section{Case Presentation}

Case 1. A 40-year-old patient (gravida 1, para 0), previously diagnosed with uterine leiomyoma and spontaneously conceived while myomectomy was planned, was admitted to the outpatient clinic on the 7 th 
week of gestation, with severe abdominal pain and vaginal bleeding. She had no systematic disease and no history of surgery. The ultrasound examination revealed a single intrauterine fetus compatible with 7 weeks and 1 day with a positive fetal heartbeat (Figure 1). A $98 \times 130 \mathrm{~mm}$ intramural fibroid located in the anterior uterine wall was also detected (Figure 2). IV hydration and analgesic treatment were recommended. After the medical treatment had failed, the surgical treatment and the termination of pregnancy options were explained to the patient. As the patient preferred the surgical treatment, myomectomy was planned after informing the patient about the potential risks related to pregnancy. Depot progesterone $500 \mathrm{mg}$ was administered intramuscularly to the patient in the preoperative period.

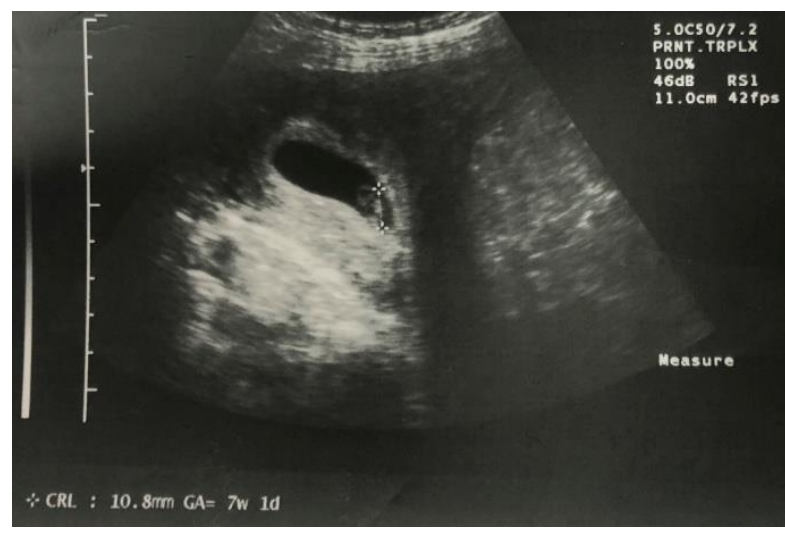

Figure 1. Intrauterine pregnancy (compatible with 7 weeks and 1 day) with positive fetal heartbeat (Case-1)

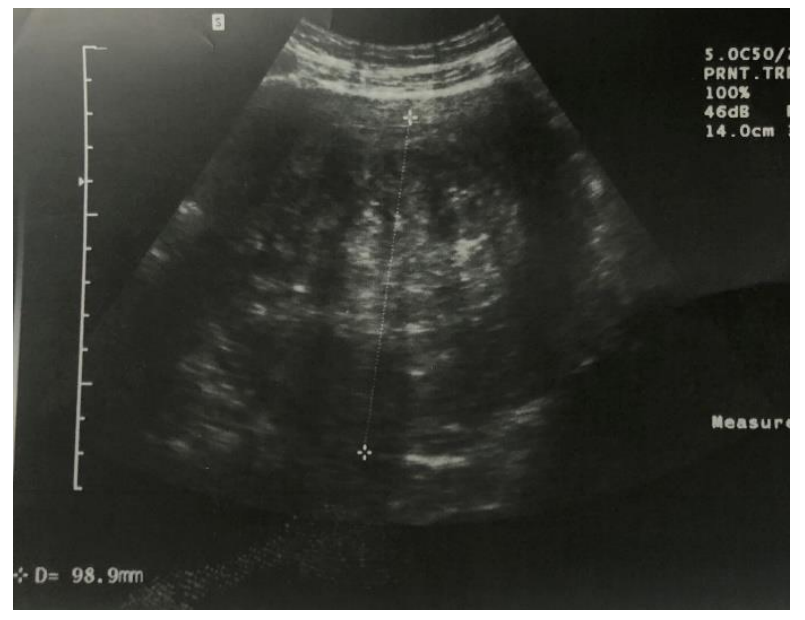

Figure 2. Fibroid of $98 \mathrm{~mm}$ in size located in the anterior uterine wall (Case-1)

Under spinal anesthesia, after the fetal heartbeat was seen sonographically, a Pfannenstiel skin incision was made. An intramural fibroid of $10 \times 14 \mathrm{~cm}$ in the anterior wall of the uterus and a subserous fibroid of $5 \times 6 \mathrm{~cm}$ in the left lateral side of the uterus were observed. The dominant myoma was shelled out through sharp dissection. The excision area was closed with 0 Vicryl and the serosa was closed with 2-0 Vicryl sutures. The subserous myoma was separated from its pedicle and excised. No bleeding and early complications occurred. Positive fetal heartbeat was confirmed by ultrasound at the end of the procedure.

The patient remained in the hospital for two days postoperatively. She had no vaginal bleeding and her hematocrit remained stable. $3 \times 200 \mathrm{mg}$ of micronized progesterone was administered vaginally during her stay. She was discharged after normal bowel movements, voiding and reassuring fetal status were seen.

The first trimester combined test was performed on the 12th week of gestation and resulted in a low risk for trisomy 21 and trisomy 13/18. The pregnancy progressed without complications. A primary Cesarean delivery was performed at 38 weeks of gestation and the patient gave birth to a healthy boy weighing $3,400 \mathrm{~g}$. Her postpartum follow-up was uncomplicated.

$\boldsymbol{C A S E} 2$. A 31-year-old patient (gravida 1, para 0) at 12 weeks of gestation according to the last menstrual period was admitted to our clinic with severe abdominal pain. She had no systematic disease and no history of surgery. A single intrauterine fetus of 12 weeks and 3 days with a positive fetal heartbeat was seen during the sonographic evaluation (Figure 3). NT was measured $1.1 \mathrm{~mm}$. The first trimester combined test was performed. An intramural fibroid of $12.5 \times 10 \mathrm{~cm}$ in size, located in the left lateral wall of the uterine corpus was observed (Figure 4). The fibroid and the placenta were on the contralateral sides of the uterus. After the conservative treatment had failed to relieve her symptoms, myomectomy was planned. Depot progesterone $500 \mathrm{mg}$ was administered intramuscularly to the patient in the preoperative period.

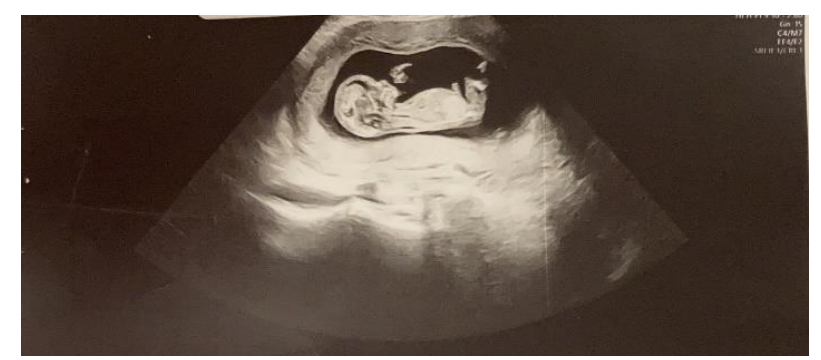

Figure 3. Intrauterine pregnancy (compatible with 12 weeks and 3 days) with positive fetal heartbeat (Case-2)

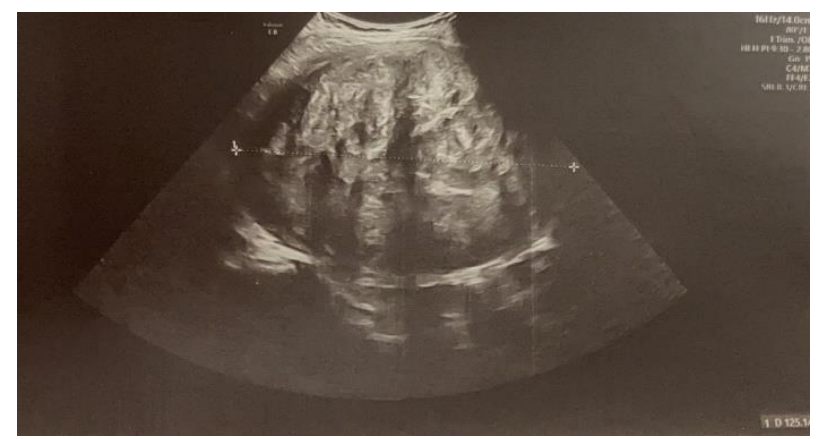

Figure 4. Fibroid of $125 \mathrm{~mm}$ in size located in the left lateral wall of the uterine corpus (Case-2) 
Under general anesthesia, after the fetal heartbeat was seen sonographically, a Pfannenstiel skin incision was made. An enlarged uterus was revealed, and a degenerated fibroid of $12 \mathrm{~cm}$ in size in the left lateral side of the uterus was observed. The enucleation of the uterine fibroid was performed, and then 0 Vicryl suture was used on the excision area. The serosa was closed with 2-0 Vicryl suture. No contact with the endometrium and the amniotic membrane was observed. After hemostasis is reassured, the procedure was concluded. Positive fetal heartbeat was confirmed through ultrasound at the end of the procedure.

The patient was administered 3x200 mg of micronized progesterone vaginally on the operation day and on the first and second days after the operation. Analgesia was provided with IV and oral paracetamol, and narcotic analgesics were not needed. The first trimester combined test resulted in low risk for trisomy 21 and trisomy 13/18. The non-invasive prenatal test, which was performed upon the request of the patient, resulted in low risk in terms of chromosomal abnormalities. On the 4th postoperative day, the ultrasound examination showed postoperative changes in the excision area (Figure 5). The fetal heartbeat was positive. The patient was discharged after normal bowel movements, voiding and reassuring fetal status were seen.

No obstetric complications were seen during her follow-up. By the time this report is written, she will have had 20 weeks of uncomplicated pregnancy.

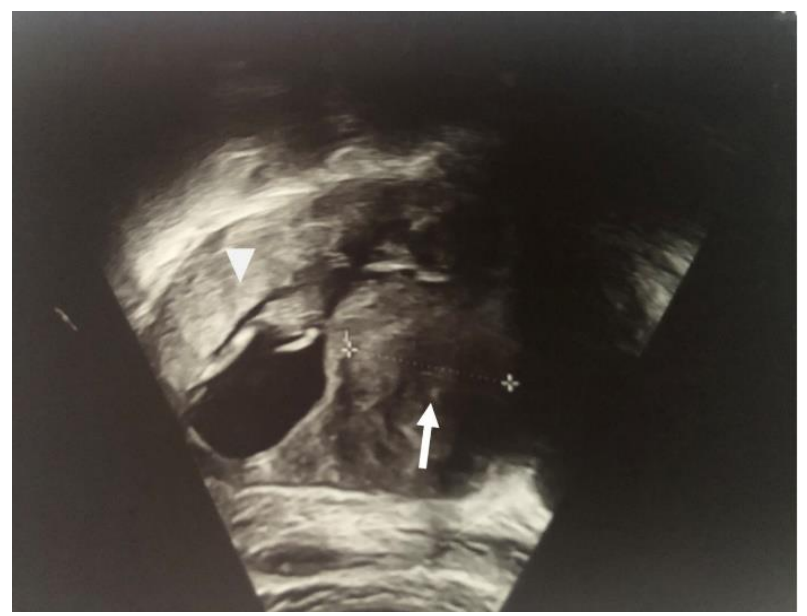

Figure 5. Ultrasound examination on the 4th postoperative day. The operation site (arrow) and the placental site (arrowhead) are seen in the contralateral sides of the uterus. (Case -2$)$

\section{Discussion}

Uterine leiomyomas are the most common benign tumors of the female genital tract. In recent years, due to late procreation, an increase has been observed in the number of cases with uterine leiomyoma during pregnancy [9].

Studies have shown that the majority of leiomyomas during pregnancy are asymptomatic $[10,11]$. However, in a small number of cases, fibroids have been associated with poor obstetric outcomes, by increasing uterine contractile activity, creating a compression effect on the growing fetus, and disrupting the blood flow of the fetus and the placenta [12]. The risk is greater especially in the presence of multiple fibroids, when the fibroid is adjacent to the placental site or when the size of the fibroid is greater than $3.6 \mathrm{~cm}\left(200 \mathrm{~cm}^{3}\right)$ [5].

The most common symptom in pregnant patients with complicated fibroids is intractable pain refractory to analgesics [13]. The pain usually presents in the first trimester or early second trimester [14]. During this period, the fibroid may increase in size [15]. Studies have shown that larger fibroids are more likely to grow; however, fibroids which are less than $5 \mathrm{~cm}$ in size are more likely to become smaller or remain the same in later periods of pregnancy. Especially, there may be an increase of $12-25 \%$ in the size of fibroids that are larger than $5 \mathrm{~cm}$ [16]. Despite this rapid growth in myoma size, insufficient blood flow causes degeneration, and degeneration causes severe pain.

The size and the vascularity of the uterus are increased during pregnancy; hence, the risk of severe hemorrhage during surgery is greater in pregnant women compared to non-pregnant individuals [17]. In these patients, adverse conditions such as severe hemorrhage requiring blood transfusion or hysterectomy, pregnancy injury and/or pregnancy loss may occur [17]. Therefore, the main treatment approach in complicated fibroids is to provide symptomatic relief to the patient with conservative treatment and to postpone the surgical intervention until after birth [14]. First-line treatment is bed rest, hydration and analgesics [18]. Most patients benefit from conservative treatment [19]. Myomectomy should be considered as the means of treatment in patients in whom symptomatic relief cannot be achieved through the applied treatment [11,20]. Except for pain, the rapid growth of myoma (doubling in size in 8 weeks), myomas that are more than $5 \mathrm{~cm}$ in size and/or myomas that are disrupting the placental site are the other indications for myomectomy [21]. In rare cases, torsion of a pedunculated myoma or bleeding of a degenerated fibroid can lead to myomectomy [22]. In our study, both patients were admitted to the outpatient clinic with severe abdominopelvic pain refractory to analgesics.

Literature reveals that myomectomy performed in the second trimester is a safe and effective treatment method in cases where the myoma is not related to the uterine cavity $[19,23]$. The outcomes of myomectomies performed in the first trimester are scant. There are not enough studies showing whether the success and the safety of this procedure are as high as in the second trimester. The traditional approach in cases with fibroids during the first trimester of pregnancy is the termination of pregnancy and performing myomectomy in a different session [8]. 
In our study, uterine leiomyoma was detected with pregnancy in both cases. Both patients had their first pregnancy, they did not have any other child, and neither of the two wanted to terminate their pregnancies. Therefore, although the treatment was not the standard treatment approach, it was accepted by the patients and the patients gave their consent to the operation despite the risks.

Mollica et al. reported a series of 106 pregnant patients with uterine fibroids [21]. In their study, pregnancy loss ( $0 \%$ vs. $13.6 \%$ ), PPROM (5.6\% vs. $22.7 \%$ ), preterm labor $(5.6 \%$ vs $21.6 \%)$ and post-Cesarean hysterectomy $(0 \%$ vs. $4.5 \%$ ) rates were lower for the patients who underwent myomectomy. According to the study, the earliest gestational age of the patients who had undergone myomectomy was 10 weeks. Unfortunately, they did not clearly state the number of operations performed in the first trimester. Therefore, the relationship between gestational age during myomectomy and obstetric outcomes of the procedure were not clearly revealed.

In another study, Carolis et al. retrospectively reported obstetric outcomes in 18 pregnant patients who had undergone myomectomy [14]. The gestational weeks of these patients varied between 6-14 weeks. 4 out of the 18 patients were operated on in the first trimester. Fibroids did not affect the placental site in any of the cases. Fibroid sizes varied between 2 and $15 \mathrm{~cm}$. One of the patients presented with severe abdominopelvic pain. The rest of the patients were operated on with the indication of rapidly growing pelvic mass. All the patients gave birth to their children by Cesarean section at term. In our study, similarly to the study conducted by Carolis et al., there was no relationship between the fibroids and the placental site in any of the patients. One of the patients gave birth to a healthy boy by Cesarean section at term. No obstetric complications have been observed in the other case during her pregnancy follow-up. Cesarean delivery is also planned in this case. There is no data in the literature about the frequency of obstetric follow-up of patients who have undergone myomectomy. However, all cases should be monitored closely due to the risks of uterine rupture and the patients should be informed about the risks.

\section{Conclusions}

Although there are studies in the literature reporting that myomectomies performed in the second trimester are beneficial in selected patients, there are insufficient data about the validity of these results for the first trimester. In cases that underwent myomectomy during the firsttrimester pregnancy, no fetal anomaly was observed due to the surgical procedure in the reported case reports; however, there are no large case series on this subject.

No obstetric complications developed due to the surgical procedure in the cases included in our study, similarly to the previously reported cases. One of the patients gave birth to a healthy boy by Cesarean section at term. Myomectomy can be an alternative treatment method in selected cases, despite the traditional approach of inducing abortion and performing myomectomy at a later date. All cases who underwent myomectomy during pregnancy should be monitored closely and these cases should be able to reach a medical institution as fast as possible. In order to make a conclusion about the safety and effectiveness of myomectomy operations performed in first-trimester pregnancies, the findings must be confirmed with further studies.

\section{Conflict of interest disclosure}

There are no known conflicts of interest in the publication of this article. The manuscript was read and approved by all authors.

\section{Compliance with ethical standards}

Any aspect of the work covered in this manuscript has been conducted with the ethical approval of all relevant bodies and that such approvals are acknowledged within the manuscript. Written informed consent was obtained from the patients for publication of this case report.

\section{References}

1. Casini ML, Rossi F, Agostini R, Unfer V. Effects of the position of fibroids on fertility. Gynecol Endocrinol. 2006;22(2):106-9. doi: 10.1080/09513590600604673

2. Santos L, Vicente L, João Nunes M, Nery I, Caetano M, Assunção N. Myomectomy in early pregnancy - A case report. Gynecol Surg. 2006;3(3):228-229. doi: 10.1007/s10397-006-0210-4

3. Qidwai GI, Caughey AB, Jacoby AF. Obstetric outcomes in women with sonographically identified uterine leiomyomata. Obstet Gynecol. 2006;107(2 Pt 1):376-82. doi: 10.1097/01.AOG.0000196806.25897.7c

4. Laughlin SK, Baird DD, Savitz DA, Herring AH, Hartmann KE. Prevalence of uterine leiomyomas in the first trimester of pregnancy: an ultrasound-screening study. Obstet Gynecol. 2009 Mar;113(3):630-635. doi: 10.1097/AOG.0b013e318197bbaf

5. Suwandinata FS, Gruessner SE, Omwandho CO, Tinneberg HR. Pregnancy-preserving myomectomy: preliminary report on a new surgical technique. Eur $J$ Contracept Reprod Health Care. 2008 Sep;13(3):3236. doi: 10.1080/13625180802075281

6. Zaima A, Ash A. Fibroid in pregnancy: characteristics, complications, and management. Postgrad Med J. 2011 Dec;87(1034):819-28. doi: 10.1136/postgradmedj2011-130319

7. Spyropoulou K, Kosmas I, Tsakiridis I, Mamopoulos A, Kalogiannidis I, Athanasiadis A, Daponte A, 
Dagklis T. Myomectomy during pregnancy: A systematic review. Eur J Obstet Gynecol Reprod Biol. 2020;254:15-24. doi: 10.1016/j.ejogrb.2020.08.018

8. Leach K, Khatain L, Tocce K. First trimester myomectomy as an alternative to termination of pregnancy in a woman with a symptomatic uterine leiomyoma: a case report. J Med Case Rep. 2011 Dec 10;5:571. doi: 10.1186/1752-1947-5-571

9. Lippman SA, Warner M, Samuels S, Olive D, Vercellini P, Eskenazi B. Uterine fibroids and gynecologic pain symptoms in a population-based study. Fertil Steril. 2003 Dec;80(6):1488-94. doi: 10.1016/s0015-0282(03)02207-6

10. Lev-Toaff AS, Coleman BG, Arger PH, Mintz MC, Arenson RL, Toaff ME. Leiomyomas in pregnancy: sonographic study. Radiology. 1987 Aug;164(2):37580. doi: 10.1148/radiology.164.2.3299488

11. Exacoustòs C, Rosati P. Ultrasound diagnosis of uterine myomas and complications in pregnancy. Obstet Gynecol. 1993 Jul;82(1):97-101.

12. Flake GP, Andersen J, Dixon D. Etiology and pathogenesis of uterine leiomyomas: a review. Environ Health Perspect. 2003 Jun;111(8):1037-54. doi: 10.1289/ehp.5787

13. Lam SJ, Best S, Kumar S. The impact of fibroid characteristics on pregnancy outcome. Am J Obstet Gynecol. 2014 Oct;211(4):395.e1-5. doi: 10.1016/j.ajog.2014.03.066

14. De Carolis S, Fatigante G, Ferrazzani S, Trivellini C, De Santis L, Mancuso S, Caruso A. Uterine myomectomy in pregnant women. Fetal Diagn Ther. 2001 Mar-Apr;16(2):116-9. doi: 10.1159/000053893
15. Milazzo GN, Catalano A, Badia V, Mallozzi M, Caserta D. Myoma and myomectomy: Poor evidence concern in pregnancy. J Obstet Gynaecol Res. 2017 Dec;43(12):1789-1804. doi: 10.1111/jog.13437

16. Ouyang DW, Economy KE, Norwitz ER. Obstetric complications of fibroids. Obstet Gynecol Clin North Am. 2006;33(1):153-69. doi: 10.1016/j.ogc.2005.12.010

17. Jhalta P, Negi SG, Sharma V. Successful myomectomy in early pregnancy for a large asymptomatic uterine myoma: Case report. Pan Afr Med J. 2016;24:1-5. doi: 10.11604/pamj.2016.24.228.9890

18. Burton CA, Grimes DA, March CM. Surgical management of leiomyomata during pregnancy. Obstet Gynecol. 1989 Nov;74(5):707-9.

19. Lolis DE, Kalantaridou SN, Makrydimas G, Sotiriadis A, Navrozoglou I, Zikopoulos K, Paraskevaidis EA. Successful myomectomy during pregnancy. Hum Reprod. 2003;18(8):1699-702. doi: 10.1093/humrep/deg318

20. Michalas SP, Oreopoulou FV, Papageorgiou JS. Myomectomy during pregnancy and caesarean section. Hum Reprod. 1995 Jul;10(7):1869-70. doi: 10.1093/oxfordjournals.humrep.a136195

21. Mollica G, Pittini L, Minganti E, Perri G, Pansini F. Elective uterine myomectomy in pregnant women. Clin Exp Obstet Gynecol. 1996;23(3):168-72.

22. Vitale SG, Padula F, Gulino FA. Management of uterine fibroids in pregnancy: recent trends. Curr Opin Obstet Gynecol. 2015 Dec;27(6):432-7. doi: 10.1097/GCO.0000000000000220

23. Celik C, Acar A, Ciçek N, Gezginc K, Akyürek C. Can myomectomy be performed during pregnancy? Gynecol Obstet Invest. 2002;53(2):79-83. doi: $10.1159 / 000052997$ 